Ann. Zootech., I974, $23(3), 253-265$.

\title{
DÉGRADATION DANS LE JABOT DU COQ ET EFFICACITÉ D'AMIDONS DE MAÏS A DIFFÉRENTS TAUX D'AMYLOSE SUR LA CROISSANCE DU POULET
}

\author{
Odette SZYLIT (1), J. DELORT-LAVAL $\left({ }^{2}\right)$ et L. P. BORGIDA $\left({ }^{2}\right)$ \\ avec la collaboration technique de \\ Françoise Balland, Michèle Fislewicz et S. Gueneau \\ Laboratoire de Recherches sur la Conservation et l'Efficacité des Aliments, \\ Centre national de Recherches zootechniques, I. N. R. A., \\ 78350 Jouy en Josas
}

RÉSUMÉ

A partir de trois amidons extraits de maïs cireux (I), normal $(\mathrm{O})$ ou d'amylomaïs $(\mathrm{Y})$, il est possible d'évaluer l'influence du taux d'amylose sur la dégradation de l'amidon dans le jabot du coq adulte et son efficacité alimentaire chez le poulet en croissance.

L'amidon de l'amylomaïs (Y) se distingue à la fois par la forme irrégulière de ses grains et par un spectre de diffraction aux rayons $\mathrm{X}$, qui l'apparente à la fécule de pomme de terre. Il est peu sensible à l'action d' $\alpha$-amylases bactérienne ou pancréatique et sa dégradation dans le jabot du coq reste limitée.

Vis-à-vis de ces mêmes enzymes, les amidons $I$ et $O$ diffèrent peu : la dégradation de l'amidon I est légèrement plus intense en présence d' $\alpha$-amylase bactérienne de $B$. subtilis et, par contre, plus faible sous l'action de l'enzyme pancréatique ou, in vivo, dans le jabot du coq.

Pour des poussins recevant entre 3 et 7 semaines un régime à base de tourteau de soja et d'amidon de maïs, le taux d'amylose le plus élevé entraîne une réduction de la vitesse de croissance et de l'efficacité alimentaire. Celles-ci sont reliées à une excrétion accrue d'amidon, une réduction du taux d'énergie métabolisable et du coefficient d'utilisation pratique de l'azote des régimes : respectivement $\mathrm{IO}, 3 \mathrm{~g} / \mathrm{j} ; 57,80 ; 39,0$ pour l'amidon $\mathrm{Y}$ contre 0,$03 ; 8 \mathrm{I}, 4 ; 55,6$ pour l'amidon X ; 0,$69 ; 80,2 ; 50,0$ pour l'amidon I.

Les aspects particuliers de la digestion d'amidons de structures variées chez le poulet et leurs répercussions sur l'efficacité alimentaire des régimes sont discutés, à la lumière de résultats d'autres travaux sur le monogastrique.

(1) Ad̉resse actuelle : Laboratoire d'Écologie microbienne, C. N. R. Z., 78350 Jouy en Josas.

( ${ }^{2}$ Adresse actuelle : Laboratoire de Technologie des Aliments des Animaux, C. N. R. Z., 78350 Jouy en Josas. 


\section{INTRODUCTION}

L'amidon est composé de 2 types de macromolécules, l'amylose et l'amylopectine (RADLEY I968) dont la proportion est une caractéristique variétale de nombreuses plantes amylacées : pois, blé, maïs (ZuBER I965). On appelle normaux des maïs à environ 25 p. Ioo d'amylose (I) amylomaïs, ceux qui en ont plus de 60 p. Ioo (Y) et cireux $(O)$ ceux qui en sont pratiquement dépourvus. A ces différents taux d'amylose sont associées des caractéristiques morphologiques et physico-chimiques particulières du grain d'amidon (MERCIER et al., r97o) ; certains auteurs (LEACH et ScHOCH, I96I) ont remarqué que la susceptibilité de l'amidon aux $\alpha$-amylases in vitro était plus faible lorsque le taux d'amylose était élevé.

Sur le plan nutritionnel, la présence dans l'aliment d'amidon pratiquement dépourvu d'amylose ne semble pas avoir de répercussion sur la croissance du Poulet (Mussel,hl, I944) ou du Porc (Hanson, I944). A taux d'amylose élevé, l'amidon de mais réduit chez le Rat 1'utilisation digestive de la matière sèche du régime et ralentit la croissance (SANDSTEDT et al., I962, BoRCHERs, I962). Aucune étude n'a encore porté sur l'efficacité de l'amidon en fonction de son taux d'amylose chez le Poulet. Or, dans cette espèce, intervient au niveau du jabot un processus particulier de dégradation des glucides (SzyLiT, I969), dont l'évolution dépend notamment des caractéristiques physico-chimiques du grain d'amidon. C'est pourquoi nous avons jugé utile, après avoir vérifié l'influence de l'origine variétale de l'amidon sur ses caractéristiques physico-chimiques, d'étudier sa digestion dans le jabot du coq adulte puis de tenter de relier ces données aux résultats de bilans et d'efficacité alimentaire chez le poulet en croissance.

\section{MATÉRIEL, ET MÉTHODES}

Nos essais portent sur des amidons extraits industriellement de 3 variétés de maïs, incorporés à des régimes équilibrés, dont ils constituent l'apport glucidique principal.

\section{1. - Caractérisation des amidons}

a) Taux d'amylose.

Les taux d'amylose des trois amidons expérimentaux, déterminés par une méthode à l'iode en milieu $\mathrm{CaCl}_{2}$ (Lespagnol, I966) sont de 2 à $3 \mathrm{p}$. Ioo pour l'amidon riche en amylopectine (amioca, O), 25 p. Ioo pour l'amidon normal I et 65 p. Ioo pour l'amidon riche en amylose (amylon, Y).

b) Spectre de diffraction.

Les deux premiers produits ont, d'après les spectres de diffraction aux rayons X (fig. I), une même structure dite de type $A$. Le dernier possède une structure pseudo-cristalline de type $B$ qui l'apparente à l'amidon de pomme de terre (CHARBONNière et al., 1968). 


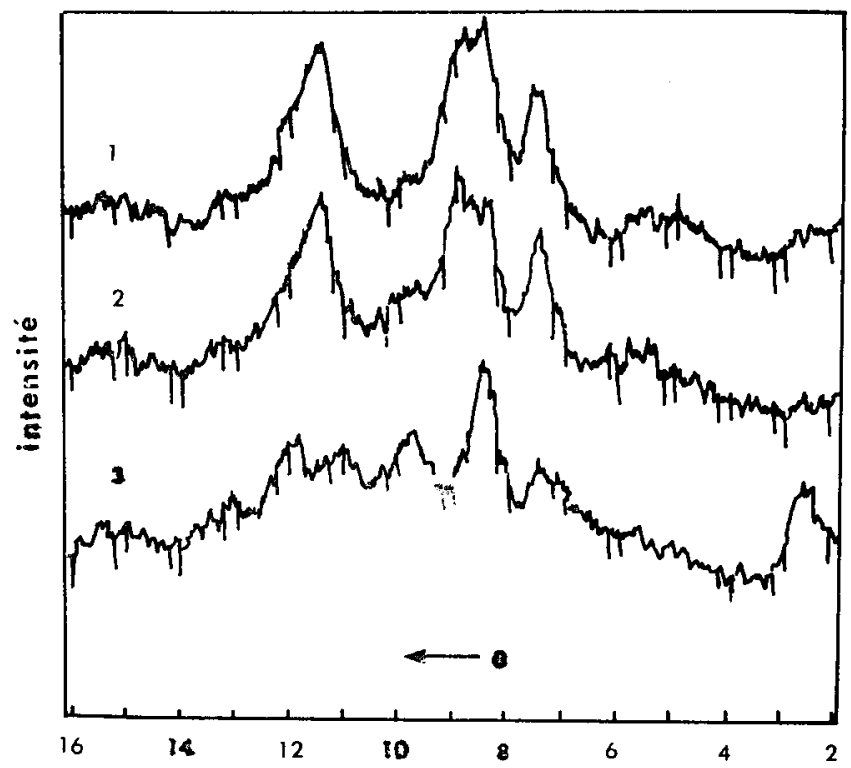

FIG. I. - Spectre de diffraction aux rayons $X$ d'amidons à taux d'amylose variable

I : amidon normal (I)
$2:$ amidon riche en amylopectine (O)
$3:$ amidon riche en amylose (Y)

c) Examen microscopique.

L'observation microscopique montre que les grains d'amidons normaux ou riches en amylopectine ont des formes semblables, bien que les seconds soient quelquefois plus anguleux. Le grain d'amidon riche en amylose se présente sous une forme plus irrégulière qui comporte parfois un long filament (fig. 2). A l'examen en solution iodo-iodurée, le taux de grains colorés apparaît faible, suggérant une influence limitée du traitement d'extraction sur l'intégrité du grain d'amidon.

\section{d) Sensibilité aux $\alpha$-amylases in vitro.}

L'attaque amylasique in vitro s'effectue à $37^{\circ} \mathrm{C}$ et à $\mathrm{pH} 6,9$ sur I g d'amidon en présence de I20 000 unités Somogyi (ANONYME 1968) d' $\alpha$-amylase bactérienne de $B$. subtilis d' $\alpha$-amylase pancréatique de porc (Sigma) ou de leur mélange isoactif. Les glucides solubles dans l'éthanol $80^{\circ} \mathrm{GL}$ qui résultent de cette réaction sont dosés par la méthode colorimétrique de Dreywood, dans les conditions de réaction décrites par LoEwus (1952). Les résultats de ces dosages sont reportés sur une courbe. Nous admettons, avec Mercier (I968), que sa pente initiale correspond à la vitesse maximale d'hydrolyse et que son ordonnée à l'origine, de la tangente à la partie approximativement linéaire de la courbe, mesure la "fraction facilement attaquable " de l'amidon soumis à l'amylolyse.

Les glucides libérés sont séparés par chromatographie sur couche mince (plaque de gel de silice G) sous 1'action successive d'un mélange propanol/acétate d'éthyle/eau I 4/2/7 (CommerForD, VANDUZEE et SCALleT, I963) durant I6 heures, puis d'un mélange méthanol/acétate d'éthyle/eau 36/52/12 (Huber, Scobell et HAN TaI 1966) durant 5 heures. Cette technique assure une bonne séparation des oligosides à moins de 8 unités glucose. Leur révélation est effectuée à l'aide du réactif de GiRI et Nigam (1953). 

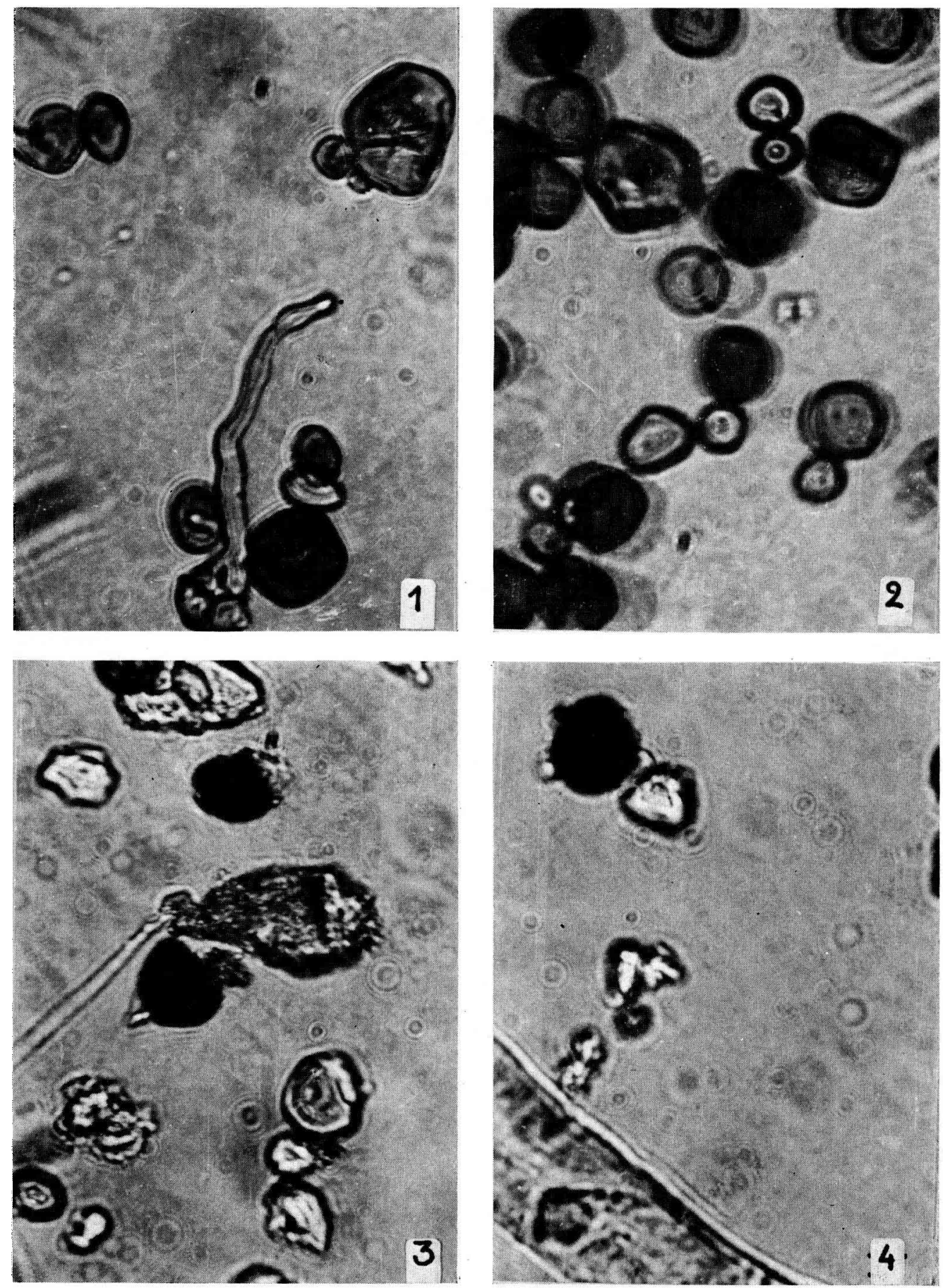

FIG. 2. - Grains d'amidon de mä̈s normal ou riche en amylose

Maïs riche en amylose : (I) aliment;

Maîs normal ou cireux : (2) aliment

(3) fèces

(4) fèces 
2. - Tests biologiques

a) Régimes alimentaires.

Les amidons de maïs expérimentaux sont incorporés dans un régime dont la composition est la suivante:

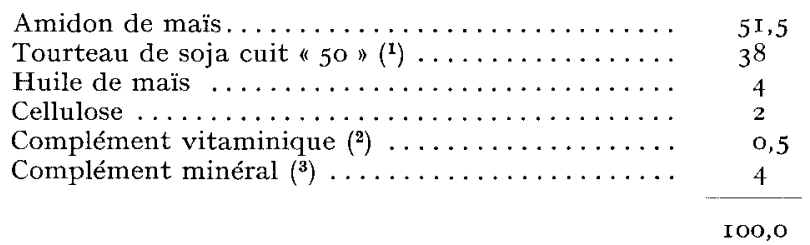

L'aliment complet est humidifié jusqu'à un taux d'environ zo p. roo, granulé à froid et desséché par passage dans une étuve à basse température $\left(70^{\circ} \mathrm{C}\right)$. Les résultats de l'analyse des trois aliments des essais de bilan et de croissance sont données dans le tableau $\mathbf{I}$.

\section{TABLEAU I}

Composition de la matière sèche des régimes expérimentaux

\begin{tabular}{l|c|c|c}
\hline \multicolumn{1}{c|}{ Régimes } & $\begin{array}{c}\text { Matières } \\
\text { minérales } \\
(\mathrm{p} .100)\end{array}$ & $\begin{array}{c}\text { Matières azotées } \\
\text { (p. 100) }\end{array}$ & $\begin{array}{c}\text { Énergie brute } \\
\text { (kcal/kg) }\end{array}$ \\
\hline & & & \\
Amioca (O) & 6,3 & 24,6 & 4532 \\
Amidon normal (I) & 6,3 & 24,7 & 4604 \\
Amylon (Y) & 6,5 & 25,8 & 4670 \\
\hline
\end{tabular}

b) Digestion de l'amidon dans le jabot du coq.

Les processus de la digestion des glucides dans le jabot sont suivis sur neuf cogs adultes, porteurs d'une fistule de cet organe (IVOREC-SzYLIT, I97I), élevés en cages individuelles et entraînés à consommer un seul repas quotidien en 3 heures. Les différents régimes sont distribués successivement à chacun des animaux, répartis en carré latin pour tenir compte des variations inđividuelles toujours très importantes. L'aliment est prélevé de façon répétée, pendant le repas, puis au cours des 5 heures qui le suivent.

Dans les contenus digestifs, les glucides alcoolosolubles totaux sont dosés par la méthode précédemment décrite et leurs concentrations rapportées à la matière sèche des échantillons prélevés.

(1) Le tourteau de soja cuit " 50 » est complété par 0,2 p. roo de L-lysine-HCl et I,8 p. roo de DL-méthionine. (Anonyme, 1972).

${ }^{(2)}$ Le mélange vitaminique (support : tourteau de soja) apporte à $100 \mathrm{~kg}$ de régime : vitamine A : I 000 ooo UI ; vitamine $D_{3}$ : I $50000 \mathrm{UI}$; vitamine $\mathrm{E}:$ I $500 \mathrm{UI}$; vitamine $\mathrm{K}_{3}: 0,59 \mathrm{~g}$; thiamine : I, I $8 \mathrm{~g}$; riboflavine : $1,76 \mathrm{~g}$; pyridoxine $: 1,76 \mathrm{~g}$; ac. nicotinique : $17 \mathrm{~g}$; ac. folique: $0,35 \mathrm{~g}$; ac. para-aminobenzoïque: $29,4 \mathrm{~g}$; inositol : $29,4 \mathrm{~g}$; panthoténate $\mathrm{Ca}: 5,88 \mathrm{~g}$; chlorhydrate de choline à $25 \mathrm{p}$. Ioo: $400 \mathrm{~g}$; vitamine $\mathrm{C}$ : 29,4 g.

(3) La composition centésimale du mélange minéral est la suivante : $\mathrm{Ca}_{3}\left(\mathrm{PO}_{4}\right)_{2}: 67,3 ; \mathrm{MgSO}_{1}, 7 \mathrm{H}_{2} \mathrm{O}$ : I $5 ; \mathrm{NaCl}: \mathrm{I}_{5} ; \mathrm{MnSO}_{4}: \mathrm{I} ; \mathrm{Fe}$ citrate : I $; \mathrm{ZnCl}_{2}: 0,24 ; \mathrm{Na}$ silicate $: 0, \mathrm{I} 5 ; \mathrm{KI}: 0,12 ; \mathrm{NaBr}: 0,075 ; \mathrm{Na}_{2} \mathrm{MoO}_{4}$, $2 \mathrm{H}_{2} \mathrm{O}: 0,06$; alun de $\mathrm{K}: 0,03 ; \mathrm{H}_{3} \mathrm{BO}_{\mathrm{g}}: 0,024 ; \mathrm{CuSO}_{4}: 0,024 \mathrm{CoSO}_{4}, 7 \mathrm{H}_{2} \mathrm{O}: 0,006 ; \mathrm{Na}_{2} \mathrm{SeO}_{3}: 0,00025$. 
c) Tests de croissance.

Les tests de croissance entre 3 et 7 semaines portent sur des poussins mâles de même souche, placés en cages individuelles et nourris ad libitum. Les trois lots expérimentaux sont constitués de I6 animaux, choisis sur la base du poids vif et du gain de poids durant la semaine préexpérimentale. On contrôle, chaque semaine et pour chaque animal, la quantité d'aliment consommée et le poids vif après un jeûne hydrique de 16 heures.

\section{d) Bilans.}

A la fin de l'essai de croissance, quatre animaux de chaque lot sont maintenus durant trois jours en cages individuelles et reçoivent une même quantité d'aliment. Les excreta, pulvérisés chaque jour d'acide sulfurique dilué au quart $(\mathrm{p} / \mathrm{p})$ sont collectés, pesés et lyophilisés le dernier jour de l'essai. Sur ces échantillons et les aliments correspondants sont déterminés la matière sèche, l'azote selon Kjeldahl, l'énergie par bombe calorimétrique et l'amidon par une méthode enzymatique à l'amylo-glucosidase (Thivend, Mercier et Guilbot, 1965). Les bilans ont été établis, sans correction, à partir de ces données.

\section{RÉSULTATS}

\section{I. - Sensibilité des amidons de maïs à l'action des $\alpha$-amylases in vitro (fig. 3 )}
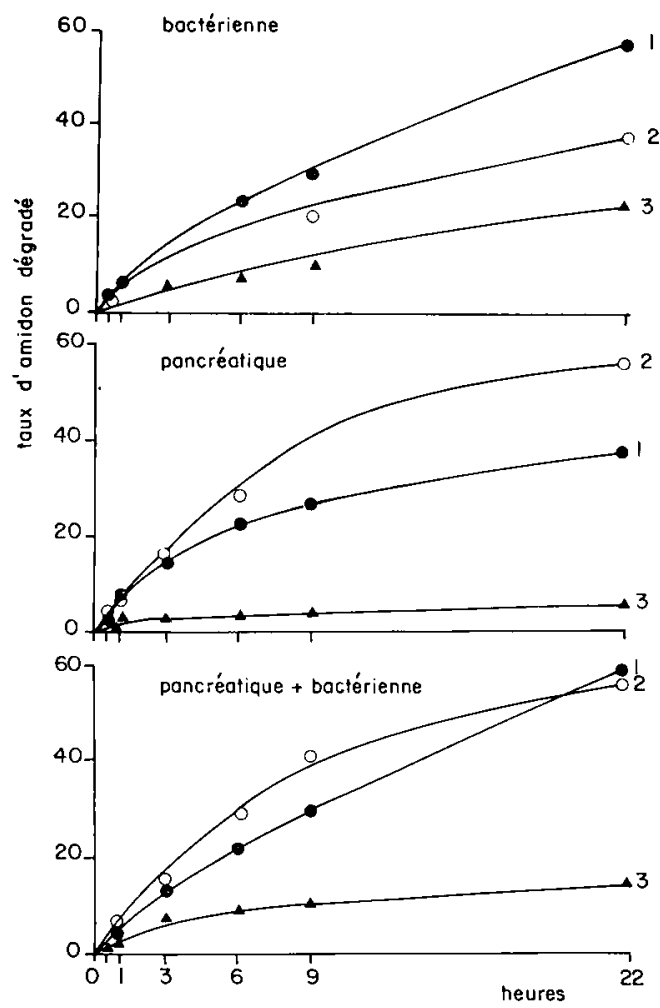

FIG. 3. - Sensibilité des amidons de mä̈s à l'action de deux $\alpha$-amylases in vitro

$$
\begin{aligned}
& \text { I : amidon riche en amylopectine } \\
& 2: \text { amidon normal (O) } \\
& 3 \text { : amidon riche en amylose (Y) }
\end{aligned}
$$


Par le test classique d' $\alpha$-amylolyse à l'enzyme bactérienne, la sensibilité des divers amidons se classe en raison inverse de leur taux d'amylose : $58-36$ et $\mathbf{r} 6 \mathrm{mg}$ de glucides alcoolosolubles apparaissent respectivement durant la première demiheure d'incubation des amidons $\mathrm{I}, \mathrm{O}, \mathrm{Y}$.

Il en va de même pour la fraction d'amidon facilement attaquable, qui est respectivement de $\mathbf{1} 6$, 9 et $\mathrm{I}$ p. roo pour ces 3 mêmes produits. Le remplacement complet de l'enzyme bactérienne par la pancréatique inverse le classement des amidons normal et riche en amylopectine. Si l'on substitue partiellement à l'amylase bactérienne une amylase pancréatique, l'amylon reste toujours le moins attaquable. Pour les deux autres amidons, les vitesses initiales et les taux de dégradation après 22 heures sont comparables.

La séparation par chromatographie sur couche mince des produits de la dégradation enzymatique des 3 amidons montre que l'action de l'enzyme pancréatique se caractérise par l'absence de glucose et par une accumulation de maltose, maltotriose et maltotétraose, la concentration de ce dernier glucide ne diminuant qu'après 9 heures d'amylolyse. L'effet de l'enzyme bactérienne s'en distingue par l'apparition très précoce ( 3 heures) de glucose et par la persistance durant les 22 heures de l'hydrolyse d'une forte concentration du maltopentaose par rapport à celle du tétraose. Comme le montre la figure 4 , la formation préférentielle du maltopentaose plutôt que du tétraose comme $\alpha$-dextrine limite paraît très caractéristique de l'enzyme bactérienne.

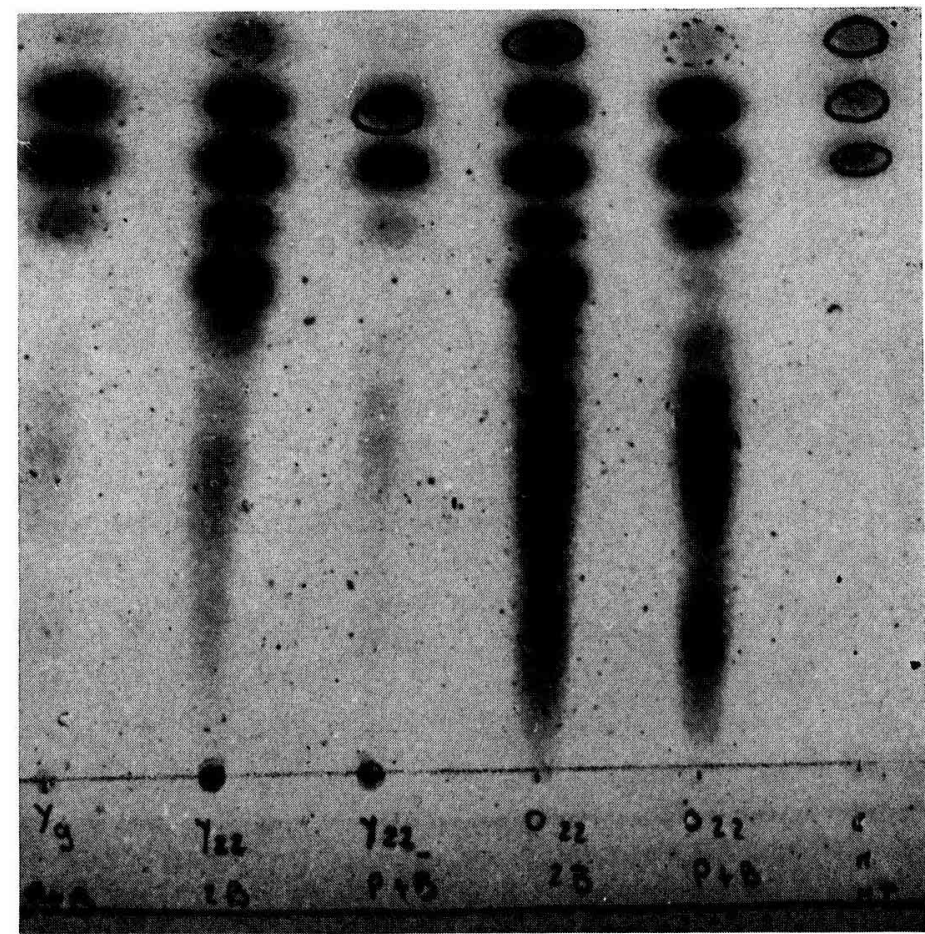

FIG. 4. - Chromatographie sur couche mince des glucides à courte châne provenant d'amidon normal (O) ou riche en amylose (Y), après 22 heures d'hydrolyse sous l'action d'amylase bactérienne (B) ou d'un mélange d'amylases bactérienne (B) et pancréatique (P). 
Le mélange de quantités isoactives des deux enzymes entraîne l'apparition de produits de dégradation dont la répartition est similaire à celle due à l'enzyme pancréatique. Il faut noter seulement la présence de traces de glucose et surtout, après 6 et 9 heures, une concentration du tétraose supérieure à celle des hydrolysats à l'amylase pancréatique seule.

\section{2. - Amylolyse in vivo dans le jabot du coq}

L'amidon est dégradé dans le jabot du coq et la concentration de glucides à courte chaîne produits augmente durant les premières heures après le repas puis diminue. Le comportement de nos trois amidons in vivo dans le jabot du coq (fig. 5) est du même type que celui observé in vitro en présence du mélange d'enzyme bactérienne et pancréatique. L'intensité de la dégradation dépend largement de la structure de l'amidon; en effet, l'amidon riche en amylose se distingue à la fois par sa vitesse d'amylolyse plus faible et par la nature des glucides alcoolosolubles formés, car trois heures après le début du repas, aucune apparition du glucose n'est encore notée. Le taux de dégradation de l'amidon riche en amylopectine reste toujours légèrement inférieur à celui de l'amidon normal.

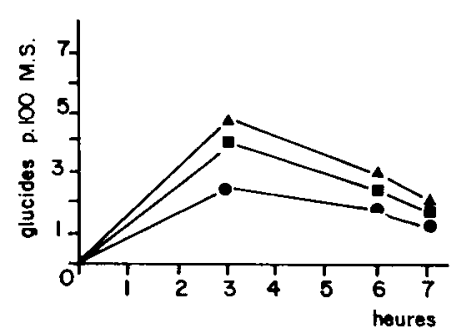

FIG. 5. - Amylolyse in vivo dans le jabot du coq adulte

Amidon de mais

- normal

- riche en amylopectine

- riche en amylose.

\section{3. - Bilans énergétiques et azotés chez le poulet en croissance}

La comparaison des bilans (tabl. 2) met en évidence le comportement très particulier des animaux soumis au régime riche en amylose. Son ingestion entraîne en effet un accroissement très sensible de l'élimination d'azote et une excrétion près de deux fois plus élevée de matière sèche et d'énergie, expliquée notamment par la présence de 25 p. Ioo d'amidon non dégradé dans les excreta du poulet.

Dans les fèces, les taux de glucides alcoolosolubles sont comparables dans tous les régimes et leur excrétion est donc approximativement doublée dans le cas des régimes riches en amylose. Avec les deux autres régimes, les bilans ne différent pas significativement, tous les critères retenus (azote fixé, concentration en énergie métabolisable, amidon fécal) étant toutefois légèrement plus favorables au régime à base d'amidon normal. 


\section{TABLEAU 2}

Bilan métabolique de l'énergie, des glucides et de l'azote (moyenne journalière de 4 animaux)

\begin{tabular}{|c|c|c|c|}
\hline Régimes & I & $\mathrm{O}$ & $\mathrm{Y}$ \\
\hline MS ingérée (g) & 91,5 & 92,0 & 91,5 \\
\hline MS excrétée (g) & 24,1 & 21,7 & 42,8 \\
\hline Amidon excrété (g) & 0,69 & 0,03 & 10,3 \\
\hline $\begin{array}{l}\text { Énergie métabolisable (p. } 100 \\
\text { énergie brute ingérée) . . . . . }\end{array}$ & 80,2 & 81,4 & 57,8 \\
\hline Matières azotées ingérées $(\mathrm{g}) \ldots$ & 22,5 & 22,6 & 23,6 \\
\hline $\begin{array}{l}\text { Coefficient d'utilisation pratique } \\
\text { de l'azote }(\mathbf{1}) \quad \ldots \ldots \ldots \ldots \ldots\end{array}$ & $50,0^{a}$ & $55,6^{a}$ & $39,0^{b}$ \\
\hline
\end{tabular}

( $\left.{ }^{1}\right)$ Les moyennes accompagnées de la même lettre ne diffèrent pas de manière significative $(\mathrm{P}<0,05)$.

\section{4. - Effucacité des régimes pour la croissance du poulet (tabl. 3)}

Malgré une ingestion de quantités comparables de matière sèche, la croissance moyenne de nos trois lots d'animaux est différente selon le régime, 1'aliment riche en amylose étant à cet égard le moins efficace. On note une adaptation des animaux au régime pauvre en amylose (I) : pendant les 2 premières semaines d'essai, ce régime ne permet qu'une croissance médiocre ( $308 \mathrm{~g}$ contre $353 \mathrm{~g}$ pour l'amidon normal), alors qu'après 4 semaines, cette différence disparaît.

\section{TABLEAU 3}

Effrcacité des régimes chez le poulet entre 3 et 7 semaines (par jour et par animal)

\begin{tabular}{c|c|c|c}
\hline \hline Régimes & $\begin{array}{c}\text { MS ingérée } \\
(\mathrm{g})\end{array}$ & $\begin{array}{c}\text { Gain de poids } \\
(\mathrm{g})\end{array}$ & $\begin{array}{c}\text { Indice de } \\
\text { consommation }\end{array}$ \\
\hline & & & \\
$\mathrm{I}$ & 55,2 & $26,9^{a}$ & $2,06^{a}$ \\
$\mathrm{O}$ & 52,1 & $27,2^{a}$ & $1,92^{b}$ \\
$\mathrm{Y}$ & 54,8 & $23,6^{b}$ & $2,24^{c}$ \\
\hline
\end{tabular}

(1) Les moyennes accompagnées de la même lettre ne diffèrent pas de manière hautement significative $(\mathrm{P}<0,01)$.

Les indices de consommation accusent encore les différences entre groupes et classent les 3 régimes dans l'ordre prévisible à partir des données du bilan. 


\section{DISCUSSION ETT CONCLUSIONS}

I,e taux d'amylose de l'amidon de maïs granulaire influe sur l'efficacité alimentaire des régimes du poulet. Les croissances plus faibles que l'on observe avec les amidons riches en amylose sont dues en partie à une diminution de la digestibilité de l'amidon et de l'azote du régime. Des observations analogues ont été faites par SANDSTEDT et al. (I962) sur le Rat.

Il nous a paru intéressant de rechercher, au niveau digestif, la raison de ces différences. Il est connu, que les caractéristiques de l'amylolyse in vitro, dépendent davantage de l'origine de l'amylase que du rapport amylose/amylopectine quand l'amidon a perdu sa structure granulaire. C'est ainsi que Tung et al. (I953), RoByt et FRENCH, (I967) et BANKs et al. (I97I) ont montré l'importance primordiale de l'origine de l'amylase sur les caractéristiques de la dégradation des chaînes d'amylose. Cet effet est d'autant plus net que le substrat amylose disparaît, indépendamment des autres paramètres de la réaction enzymatique. Des conclusions analogues ont été tirées à partir d'amidons solubles, à taux d'amylose variables, soumis à l'action d'une $\alpha$-amylase bactérienne de $B$. subtilis (Dube et Nordin, I962, BIRD et Hopkins, I954) ou salivaire (BIRD et Hopkins, I954) et pancréatique (TUNG et al, I953). Par contre, on observe, pour une enzyme donnée, que la structure granulaire peut modifier considérablement la vitesse de dégradation de l'amidon, sans agir sur la répartition des produits terminaux de 1'amylolyse. Ainsi, dans le cas d'amidons crus de maïs (SANDSTEDT et Gates, I954) et de blé (Stamberg et BaIley, I939), la vitesse de dégradation décroît avec le taux d'amylose de l'amidon.

Nos tests in vitro confirment ces résultats et montrent, de plus, qu'un mélange d'enzymes a des modalités de dégradation originales par rapport à celles de chaque enzyme prise séparément. Cette observation nous permet de comprendre que les tests d'amylolyse classique in vitro par une $\alpha$-amylase bactérienne, ne peuvent traduire que d'une manière imparfaite la dégradation de l'amidon in vivo, qui s'effectue dans le tube digestif sous l'action de plusieurs enzymes agissant conjointement. Il en va notamment ainsi dans le jabot, où l'amidon se dégrade sous l'action d'une part des amylases salivaires, dont les caractéristiques sont voisines de celles de l'amylase pancréatique, et d'autre part des enzymes bactériennes (SzYLIT, I969). Il est, dans ces conditions, intéressant de noter que, durant les premières heures du test in vitro en présence d'un mélange d'enzymes, les trois amidons se classent dans le même ordre que dans le test effectué au niveau du jabot; ce n'est qu'au-delà de 6 heures que s'observe l'attaque préférentielle de l'amidon riche en amylopectine par rapport à l'amidon normal.

Au cours de la digestion chez le Poulet, l'amylose paraît peu dégradée, puisque l'amidon qui en est riche représente $25 \mathrm{p}$. Ioo de la matière sèche excrétée. Par contre avec les deux autres régimes, l'amidon est pratiquement absent sous la forme granulaire dans les excreta. Les différences observées entre les trois amidons ne s'expliquent donc pas uniquement par celles du taux d'amylose. La structure de 1'amidon doit également intervenir et nous rappellerons à cet égard que l'amidon riche en amylose se distingue des deux autres à la fois par son spectre de diffraction aux rayons $\mathrm{X}$ qui l'apparente aux amidons de tubercule et par sa morphologie microscopique particulière (fig. 2). 
Les différences d'excrétion d'amidon et d'azote enregistrées peuvent expliquer la moindre efficacité énergétique et azotée des régimes. L'utilisation digestive de l'amidon riche en amylose est médiocre pour le Poulet. Cette plus faible digestibilité, liée à la structure de l'amidon, a déjà été constatée chez le Rat avec des régimes à base d'amylo-maïs (SANDSTEDT et al., I962, Borchers, I962). Selon BooHer et al. (I95I), des amidons à $25,50,63$ et 77 p. Ioo d'amylose sont, pour cette espèce, dégradés respectivement à $95, \mathrm{I} ; 7 \mathrm{I}, 3 ; 76,9$ et $66,0 \mathrm{p}$. Ioo. Ces mêmes auteurs démontrent également que les amidons cireux permettent une croissance et une utilisation digestive analogues à l'amidon normal.

L'efficacité médiocre de la fraction glucidique du régime à base d'amylose s'accompagne d'un moindre rendement métabolique de l'azote alimentaire, vraisemblablement lié à 1'accroissement de 1'excrétion de matière sèche et à une efficacité métabolique réduite de l'azote absorbé. A cet égard, ZELTER et al. (Ig66) soulignent que l'ingestion de fécule crue entraîne, chez le Porc soumis à un régime protéiprive, une stimulation de l'excrétion urinaire d'azote endogène $(+28$ p. IOO) et un accroissement substantiel $(+59$ p. Ioo) de la dépense métabolique fécale, qui expliquent la moindre utilisation protidique nette d'un régime à base de fécule crue, complémenté par une protéine équilibrée. Cette hypothèse pourrait être confirmée par mestre de l'excrétion d'azote chez le Poulet soumis à un régime protéiprive à base d'amylose. Toutefois, l'effet de l'amylose semble dépendre du taux protéique de la ration : dans un régime pauvre en protéines, REUSSNER et al. (1963) constatent, sur la croissance du Rat, un effet favorable du régime riche en amylose extraite du maîs par rapport à l'aliment à base d'amylopectine, moins bien accepté par l'animal. Dans un régime plus riche en azote, ils obtiennent un résultat inverse, conforme à nos propres observations. La différence d'efficacité azotée entre nos deux autres régimes $(O$ et I) n'est pas significative. Toutefois, l'intensité différente de la formation des glucides simples dans le jabot suggère que, sur les sites mêmes de la protéosynthèse tissulaire, le rapport entre azote et énergie disponible à un temps donné pourrait être plus favorable au régime à base d'amidon normal.

Quoi qu'il en soit, l'effet de la structure de l'amidon sur l'efficacité globale d'un régime suffisamment riche en protéines est très nettement mise en évidence dans cet essai. Les mécanismes responsables de ces effets, mériteraient une analyse approfondie.

Reçu pour publication en avril 1974.

\section{REMERCIEMENTS}

Nous remercions M. R. Charbonnière pour l'étude diffractométrique des amidons de maïs ainsi que la Société Roquette frères pour la fourniture gracieuse des amidons expérimentaux.

\section{SUMMARY}

STUDY OF MAIZE STARCHES WITH DIFFERENT AMYLOSE LEVELS : BREAKDOWN IN THE; COCK'S CROP AND EFFECT ON CHICKEN GROWTH

Three types of starch from waxy maize (I), normal maize (O) or high amylose maize (Y) were used to estimate the effect of the amylose level on the breakdown of starch in the crop of adult cocks and on its feed efficiency in growing chicken. 
High amylose maize (Y) starch is characterized both by the irregular form of its granules and by the fact that its $x$-ray diffraction spectrum is similar to that of potato starch. It is only little affected by the action of bacterial or pancreatic $\alpha$-amylases and its breakdown in the crop of the cock remains limited.

The behaviour of starches $\mathrm{I}$ and $\mathrm{O}$ is almost the same in the presence of the above mentioned enzymes : the breakdown of starch $I$ as compared with that of starch $O$ is a little more intense in the presence of bacterial $\alpha$-amylase from $B$. subtilis and weaker in the case of the pancreatic enzyme or, in vivo, in the cock's crop.

In 3 weeks old chicken receiving, during 28 days, a diet based on soyabean oil-meal and maize starch, the highest amylose level brought about a reduction of the growth rate and of the feed efficiency. The latter were connected to increased starch excretion, reduction of the level of metabolisable energy and of the net protein utilization : respectively, $10.3 \mathrm{~g} / \mathrm{day} ; 57.8 ; 39.0$ for $Y$ starch versus $0.03 ; 8 \mathrm{I} .4 ; 55.6$ for $\mathrm{O}$ starch and $0.69 ; 80.2 ; 50.0$ for I starch.

Particular aspects of the digestion in chicken of starches of various structures and of their repercussions on the feed efficiency of the diets are discussed as compared to data from studies on monogastric animals.

\section{RÉFÉRENCES BIBLIOGRAPHIQUES}

Anonyme, 1972. Recommandations techniques en alimentation animale. Doc. Tech. I7I A. E. C. Commentry.

ANonyme, I968. The determination of amylase in serum, urine and other fluids. Tech. Bull $\mathrm{n}^{0} 700$, Sigma, St-Louis. Mo.

Banks W., Mazumder N. K., Srooner R. L., I971. Studies on the starch degradating enzymes of bovine serum. Comp. Biochem. Physiol., 40 B, 983-99o.

Bird R., Hopkins R. H., I954. The action of some $\alpha$-amylases on amylose. Biochem. J., 56, 86-99.

Boohlr L. E., BEhan I., MCMeans E., 195I. Biological utilization of unmodified starches. $J$. Nutr., 45, 75-95.

Borchers R., 1962. A note on the digestibility of starch of high amylose corn in rats. Cereal Chem., 39, I45-I46.

Charbonnik̀re R., Mercier C., Tollikr M. T., Gullbot A., ig68. lítude diffractométrique des amidons de maïs à teneur variable en amylose. Stärke, 20, 75-78.

Commerford J. D., Vanduzee G. T,, Scallet B. L., ig63. Macropaper chromatography of corn starch hydrolysate. Cereal Chem., 40, $482-486$.

Dure, S. K., Nordin P., ig62. The action pattern of sorghum $\alpha$-anylase. Arch. Biochem. Biophys., 99, $105-108$.

Grirt K. V., Nigam V. N., 1953. Separation of simple saccharides and oligosaccharides by circular paper chromatography. Naturveiss., 40, 343-344.

Hanson L. E., I944. Waxy corn us non waxy corn for pigs in dry lot. Neb. Agr. Exp. St. Ann. Rep., $\mathbf{5 7}$, 66, cité par Hixon R. M., r968, in Radefy J. A. Starch and its derivates, 260. Chapman and Hall ed., Londres.

Huber C. N., Scobril. H., Han Ta1, ig66. Determination of corn syrup by direct densitometry of thin layer chromatography. Cereal Chem., 43, 342-346.

Ivorec-Szylit O., i971. Note sur la fistulation du jabot du coq. Ann. Biol. anim. Bioch. Biophys., 11, $733-735$.

Leach M. W., Schocr T. J., Ig6r. Structure of the starch granule. II : Action of various amylases on granular starches. Cereal Chem., 38, 34-46.

Lespagnol V., 1966. Dosage de l'anylose. C. R. stage au Laboratoire de la Société Roquette Frères.

Lowwus F. A., 1952. Improvement in anthrone method for determination of carbohydrates. Anal. Chem., 24, 219.

Mercier C., I968. Contribution à l'étude de la structure du grain d'amidon au moyen de méthodes physiques et enzymatiques. Thèse Doct. d'État. No C. N. R. S., A. O. 2413.

Mercier C., Charbonnitere R., Gallant D., Guilbot A., ig7o. Évolution de quelques caractéristiques des amidons extraits de grains de maïs normal et d'amylomaïs au cours de leur formation. Stärke, 22, 9-I6.

Mussenl F. E., I944. Growth promoting value for chicks of waxy corn. Neb. Agr. Exp. St. Ann. Rep., 57, 79, cité par Hixon R. M., I968, in RADLEY J. A., Starch and its derivates, 260, Chapman and Hall ed., Londres.

RAdLey J. A., I968. Starch. and its derivates, Chapman and Hall ed., Londres, 12-65.

Reussner G., Andros J., Thiessen R., 1963. Studies on the utilization of various starches and sugars in the rat. J. Nutr., 80, 248-29r. 
RoByt J. F., FRENCh D., I967. Muitiple attack hypothesis of $\alpha$-amylase action : action of porcine pancreatic, human salivary and A spergillus oryzae $\alpha$-amylases. Arch. Biochem. Biophys., 122, 8-I6.

Sandstedt R. M., Gates R. L., I954. Raw starch digestion : a comparison of the raw starch digesting capacities of the amylases systems from $4 \propto$-amylases sources. Food Research, 19, I90-I 99 .

Sandstedt R. M., Strahan D., Ueda S., Abott R. C., I962. The digestibility of high amylose corn starch compared to that of other starches. The apparent effect of the ae gene on susceptibility to amylase action. Cereal Chem., 39, $123-145$.

StamberG D. E., Bailey C. M., 1939. Studies on wheat starch. The action of amylase on raw wheat starches. Cereal Chem., 16, 3I9-335.

Szylit O., rg69. Physiologie de la digestion des glucides dans le jabot du coq. VIII Cong. Intern. Nutr., Prague, résumé comm., $57 \mathrm{I}$.

Thivend P., Mercier C., Guilbot A., ig65. Dosage de l'amidon dans les milieux complexes. Ann. Biol. anim. Bioch. Biophys., 5, 5I3-536.

Tung J. İ. K., Banraman M., Caldwell M. L., I953. A comparison of the action of several $\alpha$-amylase upon a linear fraction from corn starch. J. Am. Chem. Soc, 75, 5548-5554.

Zelter S. Z., Charlet-Lery G., Delort-Laval J., ig66. Influence de la fécule crue et cuite de pomme de terre sur les dépenses azotées métaboliques et endogènes et sur la valeur nutritionnelle d'un mélange équilibré de protéines chez le porc en croissance. C. R. Acad. Agr. France, 567-573.

Zuber M. S., i965. in Whistler L. R. et Paschall E. F., Starch chemistry and technology, 1, 43-6z, Acad. Press ed., Londres. 